\title{
October 2016 Pulmonary Case of the Month
}

\author{
Coya $T$ Lindberg, $B S^{1}$ \\ Ryan $R$ Nahapetian, $M D^{2}$ \\ F Zahra Aly, MD, PhD, FRCPath \\ ${ }^{1}$ University of Arizona College of Medicine Tucson, Tucson, AZ. \\ ${ }^{2}$ Division of Pulmonary, Allergy, Critical Care, and Sleep Medicine, University of \\ Arizona, Tucson, AZ \\ ${ }^{3}$ Brody School of Medicine at East Carolina University, NC.
}

A 49-year-old man presented with chest discomfort to an outside medical facility in Arizona. He was previously healthy and had no chronic medical diseases. Physical examination was unremarkable and he was afebrile. A chest X-ray was performed (Figure 1).

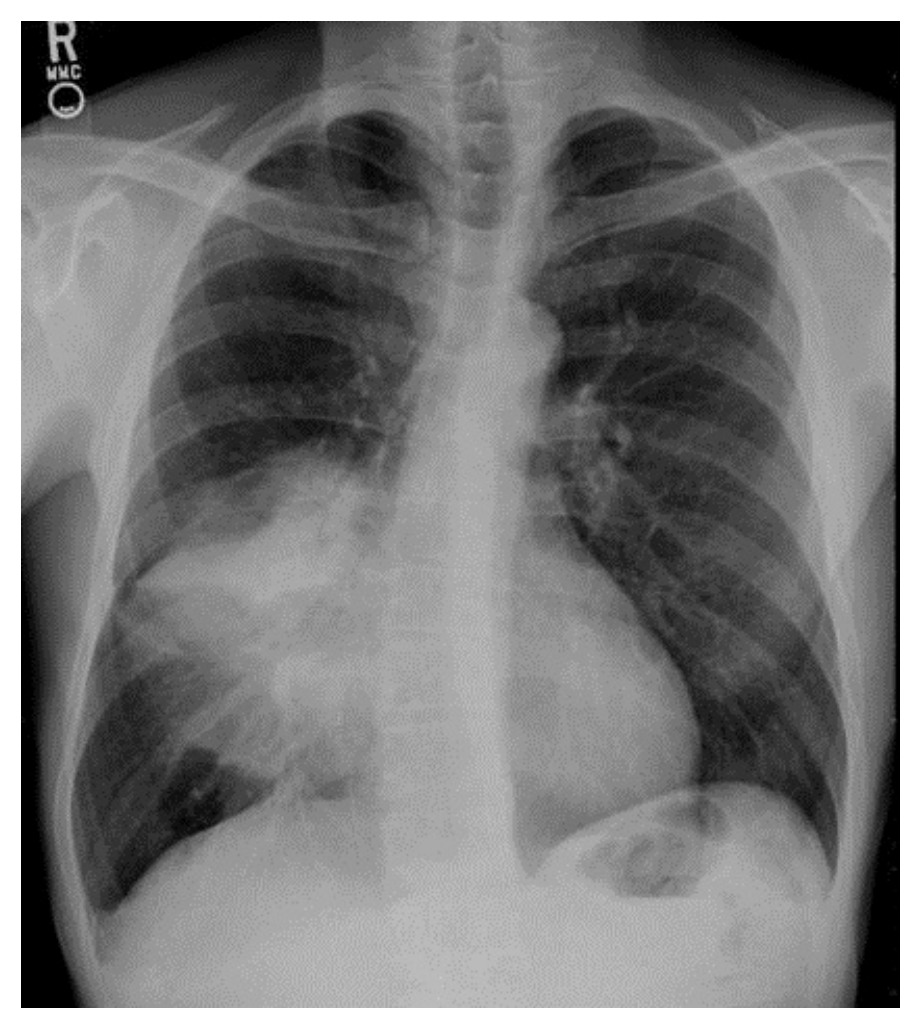

Figure 1. Initial chest x-ray

Which of the following is most likely?

1. There is a large right chest mass

2. There is a loculated pleural effusion in the minor fissure

3. There is a right ventricular aneurysm

4. There is right lower lobe consolidation

5. There is right middle lobe consolidation 


\section{Correct!}

\section{There is right middle lobe consolidation}

The lesion is in the right chest obscuring the right heart border. There are air bronchograms present indicating that this is most likely consolidation. This could be a large right chest mass although this is less likely than consolidation especially with air bronchograms present. A right ventricular aneurysm of this size would be very unusual. Antibiotics failed to resolve the lesion, although his chest discomfort resolved.

Which of the following should be performed next?

1. Bronchoscopy

2. Video-assisted thorascopic surgery (VATS)

3. Coccidioidomycosis serology

4. 1 and 3

5. All of the above 


\section{Correct!}

\section{1 and 3}

Coccidioidomycosis is endemic in Arizona and causes as much as $24 \%$ of communityacquired pneumonia (1). This patient's serology was negative but it is important to remember that serology can be falsely negative especially early in the course of disease (1). The absence of fever and other symptoms such as productive cough and dyspnea suggests that this may be something other than typical pneumonia. A course of antibiotics is not unreasonable but the patient responded minimally to the therapy. Subsequently, he underwent bronchoscopy and needle biopsy. Cytology showed atypical lymphoid cells. Flow cytometry expressed CD-19 and CD-20, lambda light chains, and weak BCL-2. Diagnosis was established as marginal zone mucosa associated lymphoid tissue (MALT) lymphoma. Five weeks of rituximab had no effect on tumor size. He had since been observationally followed with routine thoracic CT scans, with his lymphoma remaining stable and asymptomatic.

Eleven years after his initial presentation he was seen complaining of dyspnea and new-onset palpitations. His physical examination showed stable vital signs with decreased breath sounds in the right chest. Troponins and electrocardiography were unremarkable. A chest radiograph was performed (Figure 2).

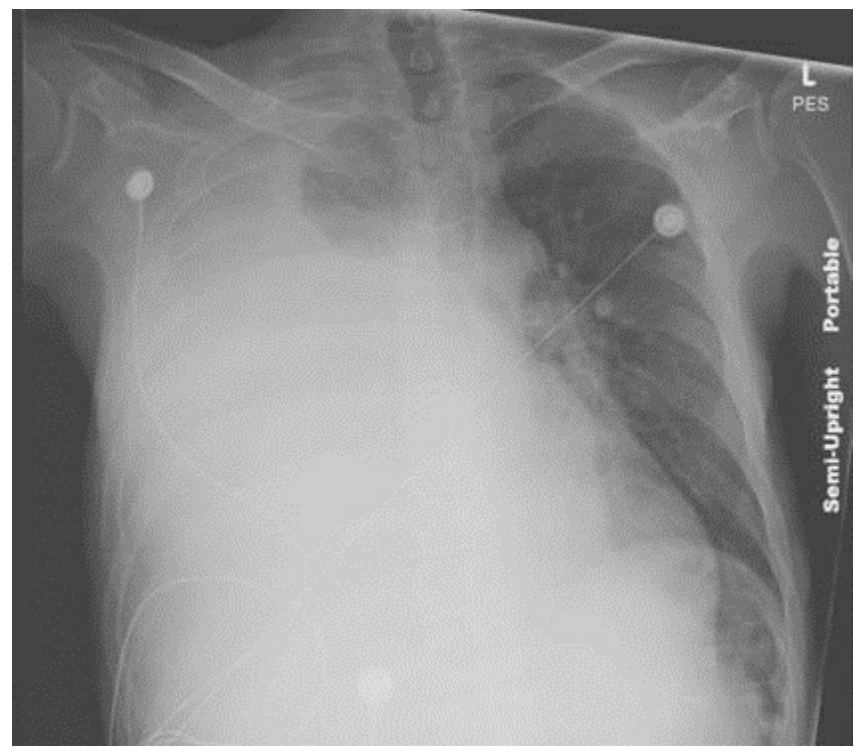

Figure 2. Portable chest x-ray taken 11 years after his initial presentation.

What of the following should be done next?

1. Thoracic CT scan

2. Bronchoscopy

3. Thoracentesis

4. 1 and 3

5. All of the above 


\section{Correct!}

\section{1 and 3}

The chest $\mathrm{x}$-ray shows a large right pleural effusion. This is shown by the volume expansion of the right chest with a shift of the trachea to the left. A collapsed right lung shows volume loss. The right pleural effusion was confirmed by a thoracic CT scan (Figure 3).

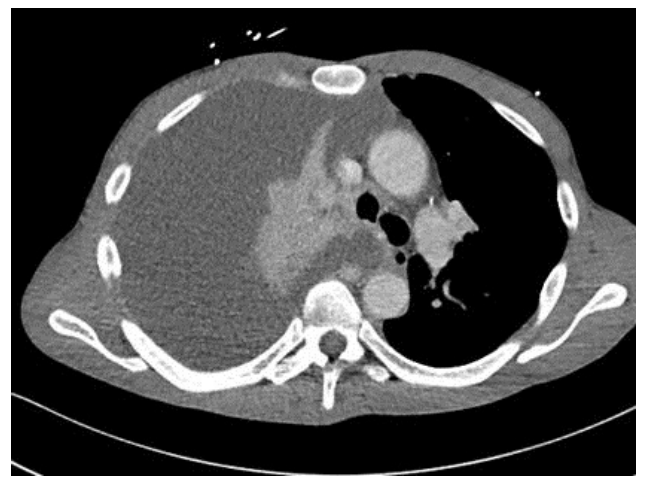

Figure 3. Representative image from thoracic CT scan in soft tissue windows showing large right pleural effusion.

Thoracentesis was performed, with $2000 \mathrm{ml}$ of serosanguinous fluid drained and sent for analysis. The fluid was found to be exudative and lymphocyte-predominant (analysis: WBC 9000 cells $/ \mathrm{mm}^{3}$, RBC 201000 cells $/ \mathrm{mm}^{3}$, PMN 11\%, Lymphocytes 82\%, Eosinophils 0\%, Mono/Macrophages 7\%, LDH 388 IU/L (vs serum LDH 131 IU/L), Protein $7.4 \mathrm{~g} / \mathrm{dL}$ (vs serum protein $6.9 \mathrm{~g} / \mathrm{dL}$ )). Plasma cells made up $35 \%$ of the lymphocyte count. Cytology showed atypical lymphocytic cells with marked nuclear enlargement and membrane irregularities (Figure 4).

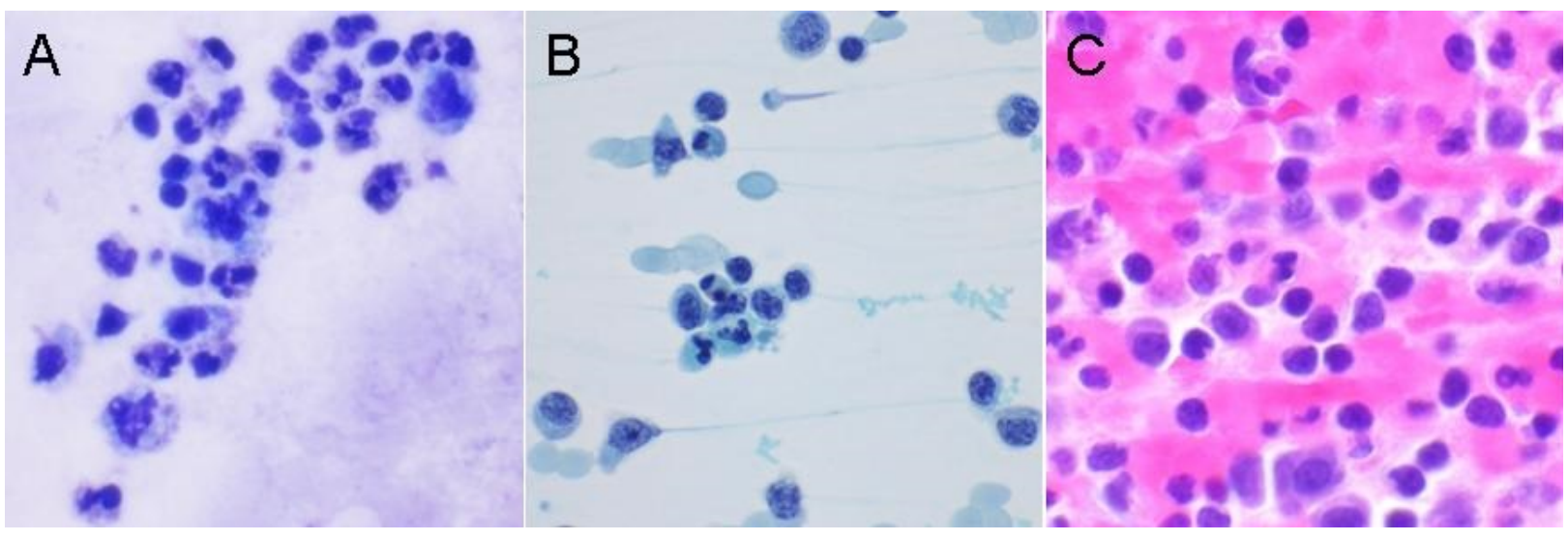

Figure 4. Pleural fluid showing atypical lymphocytic cells as seen in different preparations. A: Diff Quick (x600). B: Papanicolau (x600). C: Cell block (x600). The cells show marked nuclear enlargement and membrane irregularities.

Polymorphonuclear leukocytes in the background allow for estimation of nuclear enlargement. 
Which of the following is the most likely diagnosis that explains the pleural effusion?

1. Adenocarcinoma of the lung

2. Congestive heart failure

3. Empyema

4. MALT lymphoma

5. Small cell carcinoma of the lung 


\section{Correct! \\ 4. MALT lymphoma}

The history of MALT lymphoma combined with the exudative pleural effusion and the atypical lymphocytic cytology make this the most likely diagnosis. Congestive heart failure should present with a transudative effusion and an effusion this large limited to the right chest would be unusual. Empyema should present with a neutrophilic predominant effusion and metastatic carcinoma of the lung should present with compatible cytology showing cancer cells in the pleural fluid.

Although mucosa associated lymphoid tissue (MALT) lymphoma represents the most common type of primary pulmonary lymphoma (PPL), PPLs only make up $0.5 \%$ of primary lung malignancies (2). Pulmonary MALT lymphomas most commonly presents with either non-specific symptoms or asymptomatically. Patients are asymptomatic in $37-54 \%$ of cases $(2,4,5)$, with discovery upon routine imaging $(2,3)$. Of those with symptoms, cough or exertional dyspnea are most common, in $21-46 \%$ (3-5). Weight loss occurs in $16-23 \%(3,4) .10-25 \%$ of patients have fever, pleuritic chest pain, fatigue, or hemoptysis $(3,5)$.

Commonly reported radiographic features of pulmonary MALT lymphomas include focal lung nodules in $85-90 \%(2,3)$, with normal or dilated air bronchograms seen in $50-100 \%$ (2-6). Both solitary and multifocal lesions are common, and bilateral involvement is seen in approximately $20 \%$ (3-6). Other presentations include as round opacities (19$62 \%)(3-6)$, or as diffusely interstitial opacities (8\%) (3). Mediastinal or hilar lymphadenopathy is reported in $15-25 \%(2-6)$. Rarely, an associated pleural effusion is present, in $2-15 \%$ of reports (2-6).

Diagnosis requires confirmation by flow cytometry and/or cytology with immunohistochemistry on cell block specimen. The immunohistochemistry aids differentiation of MALT lymphoma from the other varieties of non-Hodgkin's lymphoma (2). MALT lymphomas generally follow an indolent course, and survival at five years is 93\% (2).

This case of pulmonary MALT lymphoma causing a unilateral pleural effusion is unusual. Most pulmonary MALT lymphomas are clinically asymptomatic and radiographically nonspecific. Thus, consideration of pulmonary MALT Iymphoma is appropriate when faced with nonspecific symptoms and a variety of chest imaging findings.

\section{Acknowledgments}

Carr, Gordon MD. Division of Pulmonary, Allergy, Critical Care, and Sleep Medicine, University of Arizona, Tucson, AZ 


\section{References}

1. Galgiani JN, Ampel NM, Blair JE, et al. 2016 Infectious Diseases Society of America (IDSA) clinical practice guideline for the treatment of coccidioidomycosis. Clin Infect Dis. 2016 Sep 15;63(6):e112-46. [CrossRef] [PubMed]

2. Cardenas-Garcia J, Talwar A, Shah R, Fein A. Update in primary pulmonary lymphomas. Curr Opin Pulm Med. 2015 Jul;21(4):333-7. [CrossRef] [PubMed]

3. Cordier JF, Chailleux E, Lauque $\mathrm{D}$, et al. Primary pulmonary lymphomas. A clinical study of 70 cases in nonimmunocompromised patients. Chest. 1993 Jan;103(1):2018. [CrossRef] [PubMed]

4. Huang H, Lu ZW, Jiang CG, Li J, Xu K, Xu ZJ. Clinical and prognostic characteristics of pulmonary mucosa-associated lymphoid tissue lymphoma: a retrospective analysis of 23 cases in a Chinese population. Chin Med J (Engl). 2011 Apr;124(7):1026-30. [PubMed]

5. Sui X, Song W, Jin ZY, Feng RE, Cui QC, Xue HD, Li S, Sun H. Clinical and imaging manifestations of pulmonary mucosa-associated lymphoid tissue lymphoma. Zhongguo Yi Xue Ke Xue Yuan Xue Bao. 2012 Feb;34(1):41-5. [PubMed]

6. Qiang J, Qi PF, Gao WQ, Chen DS, Yü W. Imaging findings of non-contrast CT scan, enhancement and HRCT of pulmonary mucosa-associated lymphoid tissue lymphoma. Zhonghua Yi Xue Za Zhi. 2013 Jun 4;93(21):1634-6. [PubMed] 